\title{
Not an open cluster after all: the NGC 6863 asterism in Aquila ${ }^{\star}$
}

\author{
C. Moni Bidin ${ }^{1}$, R. de la Fuente $\operatorname{Marcos}^{2}$, C. de la Fuente Marcos², and G. Carraro 3,4 \\ 1 Departamento de Astronomia, Universidad de Concepcion, Casilla 160-C, Concepcion, Chile \\ 2 Suffolk University Madrid Campus, C/ Viña 3, 28003 Madrid, Spain \\ 3 European Southern Observatory, Alonso de Cordova 3107, Vitacura, Santiago, Chile \\ e-mail: gcarraro@eso.org \\ 4 Dipartimento di Astronomia, Universitá di Padova, vicolo Osservatorio 3, 35122, Padova, Italy
}

Received 25 June 2009 / Accepted 2 December 2009

\begin{abstract}
Context. Shortly after birth, open clusters start dissolving; gradually losing stars into the surrounding star field. The time scale for complete disintegration depends both on their initial membership and location within the Galaxy. Open clusters undergoing the terminal phase of cluster disruption or open cluster remnants (OCRs) are notoriously difficult to identify. From an observational point, a combination of low number statistics and minimal contrast against the general stellar field conspire to turn them into very challenging objects. To make the situation even worst, random samples of field stars often display features that may induce to classify them erroneously as extremely evolved open clusters.

Aims. In this paper, we provide a detailed study of the stellar content and kinematics of NGC 6863, a compact group of a few stars located in Aquila and described by the Palomar Observatory Sky Survey as a non existent cluster. Nonetheless, this object has been recently classified as OCR. The aim of the present work is to either confirm or disprove its OCR status by a detailed star-by-star analysis.

Methods. The analysis is performed using wide-field photometry in the UBVI pass-band, proper motions from the UCAC3 catalogue, and high resolution spectroscopy as well as results from extensive $N$-body calculations.

Results. The spectra of the four brightest stars in this field clearly indicate that they are part of different populations. Their radial velocities are statistically very different and their spectroscopic parallaxes are inconsistent with them being part of a single, bound stellar system. Out of the four stars, only two of them have similar metallicity. The color magnitude diagram for the field of NGC 6863 does not show any clear signature typical of actual open clusters. Consistently, spatial scan statistics confirms the absence of any statistically significant, kinematically supported over-density at the purported location of NGC 6863.

Conclusions. Our results show that the four brightest stars commonly associated to NGC 6863 form an asterism, a group of nonphysically associated stars projected together, leading to the conclusion that NGC 6863 is not a real open cluster.
\end{abstract}

Key words. open clusters and associations: individual: NGC 6863 - open clusters and associations: individual: NGC 1901 open clusters and associations: general - Galaxy: evolution

\section{Introduction}

Most if not all the field stars that we observe on any given galaxy are thought to have been formed in star clusters or associations (see e.g. Lada \& Lada 2003). In the Milky Way disk and as soon as they come into existence these objects become dissolving clusters, gradually losing stars into the general field population. Open clusters are known to disintegrate on a time scale which depends both on their membership and galactocentric distance. The eventual residue of the evolution of an open cluster is often called open cluster remnant (hereafter OCR). Early $N$-body simulations gave us a first glimpse on how the final stage in the life of an open cluster may look like. For simulated systems with some 25 to 250 stars, von Hoerner (1960, 1963), Aarseth (1968) and van Albada (1968) suggested that the final outcome in the evolution of an open star cluster is one or more tightly bound binaries (or even a hierarchical triple system). Van Albada pointed out several observational candidates ( $\sigma$ Ori, ADS 12696, $\rho$ Oph, $1 \mathrm{Cas}, 8 \mathrm{Lac}$ and $67 \mathrm{Oph}$ ) as being OCRs and Wielen (1975) indicated yet another one, the Ursa Major cluster (Collinder 285).

^ Based on observations carried out at ESO La Silla, under program 281.D-5054.
Regrettably, these early numerical results failed to spur interest among the observational community and trigger any systematic study aimed at identifying open clusters undergoing the terminal phase of cluster disruption: the OCR stage. We had to wait until the late 80 s to find the first attempt. Using objectiveprism plates, Lodén (1987, 1988, 1993, and references therein) investigated the frequency of clusters in the Milky Way under the hypothesis that their stars should have similar luminosities and spectral types. He found that about $30 \%$ of the objects in his sample could be catalogued as a possible type of cluster remnant. Unfortunately, without proper kinematic information, this approach produces non conclusive results. There are known regions in the sky with many stars of similar spectral type but in which it is difficult to find two stars with the same velocity. These are some type of asterism: their stars are all at different distances and move in different directions but perspective lines them up in the sky to become optical groupings. The situation is analogous to optical doubles and binary stars. New simulations (de la Fuente Marcos 1997, 1998) confirmed early numerical results characterizing OCRs as sparsely populated stellar aggregates, rich in binaries and higher multiplicity systems and poor in low-mass members. Another main conclusion of these simulations was the intrinsically specific stellar content 
of remnants coming from clusters with very different initial populations. Open clusters originally hosting relatively small numbers of stars (perhaps not more than $10^{3}$ ) still arrive to the terminal phase of cluster evolution with a significant population of early-type, luminous stars. Enhanced preferential retention of massive stars and binaries is mainly the result of dynamically induced mass segregation. In contrast, originally rich clusters (e.g. $N>10^{4}$ ) can survive for several Gyr and when they arrive to the twilight of their dynamical lives, the brightest stars still in the main sequence are late $F$ or early $G$ spectral types. In other words, initially richer clusters remain bound for longer time and therefore the main sequence tunoff point is fainter by the time that they dissolve. This fact makes OCRs from these clusters much more difficult to identify than those from smaller clusters.

Unconnected to Lodén's work, a new list of OCR candidates was produced by Bica et al. (2001); their members being selected based on stellar density contrast considerations. Unfortunately, subsequent analyses including positional, kinematic, photometric and spectroscopic information failed to confirm even the cluster status of some of the suggested candidates (Villanova et al. 2004a; Carraro et al. 2005). Again, negative results pointed out an already well known fact, that these objects are notoriously difficult to identify. This relatively large fraction of false positives strongly hints that random samples of field stars often display features that may induce to classify them erroneously as extremely evolved open clusters. In spite of their intrinsically challenging nature, the observational search for OCR candidates has become a subject of considerable interest as it is one of the keys to understand the origin of the field star populations of the Galactic disk (see Carraro 2006).

In this research, we provide a detailed study of the stellar content and kinematics of NGC 6863, a compact group of a few stars in Aquila described by the Palomar Observatory Sky Survey as a non existent cluster. However, this object has been recently classified as OCR (Bica et al. 2001; Pavani \& Bica 2007). The aim of the present work is to either confirm or disprove this result. In this paper, we present new CCD UBVI photometry and single epoch spectroscopy, which we combine with proper motions from the UCAC3 (Zacharias et al. 2009) catalogue in an attempt to unravel the true nature and dynamical status of this object. This paper is organized as follows: in Sect. 2 we provide a historical overview of the data previously available for this object. Observations, data reduction, and overall results are presented in Sect. 3. In Sect. 4 we focus on proper motions. The statistical significance of the results is discussed in Sect. 5. In Sect. 6 we draw our conclusions.

\section{NGC 6863 in perspective}

This asterism or group of a few stars in Aquila was first identified in 1827 by Herschel (1833) as h 2065 using a 18.7 inches telescope. The same group of eight Galactic stars was included as object GC 4542 by Herschel (1864) in his "Catalogue of Nebulae and Clusters of Stars". The "New General Catalogue of Nebulae and Clusters of Stars" (Dreyer 1888) first used the name NGC 6863, describing the object as a very much compressed, small cluster including stars from the 19th magnitude downwards. No further references to this object are found until the publication of "The Revised New General Catalogue of Nonstellar Astronomical Objects" (Sulentic \& Tifft 1973). This catalog is a modern, revised, and expanded version of the original NGC. Besides incorporating the many corrections to the NGC found over the years, each object was verified on Palomar Observatory Sky Survey (POSS) prints and on plates

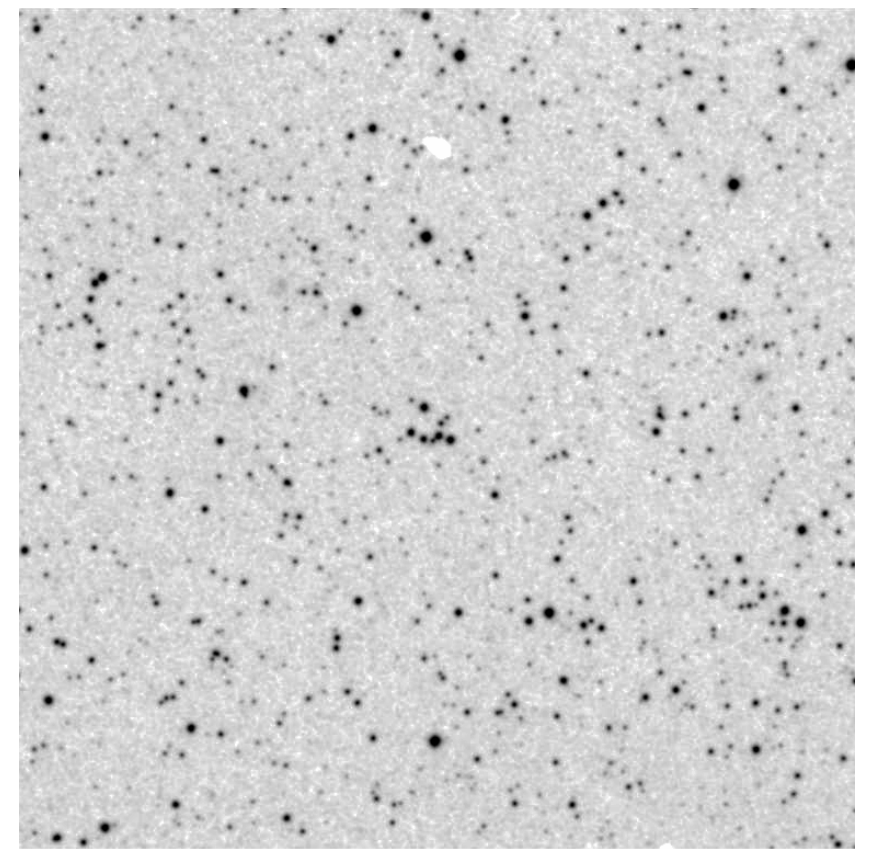

Fig. 1. Palomar Observatory Sky Survey (POSSI) image of the area covered by the present study. North is up, East to the left, and the image is 12.9 arcmin on a side. This 12 min digitized plate was acquired by the Palomar 48-in Schmidt camera using the red (O) filter on 1951.50678. The frame displayed has been processed using square root scaling.

for southern objects specifically taken for the purpose. In this catalog NGC 6863 is described as non existent. The POSSI red (O) plate is displayed in Fig. 1. The object is widely regarded as an asterism among the amateur astronomical community (see, e.g., Allison 2006) but it is listed as a bona fide cluster in both the Open Cluster Database ${ }^{1}$ (WEBDA) and the New Catalogue of Optically Visible Open Clusters and Candidates ${ }^{2}$ (NCOVOCC, Dias et al. 2002). These databases are widely used in professional open cluster studies. The latest update of WEBDA includes coordinates, $\alpha=20^{\mathrm{h}} 05^{\mathrm{m}} 07^{\mathrm{s}}, \delta=-03^{\circ} 33^{\prime} 18^{\prime \prime}, l=$ $38.278, b=-17^{\circ} .996$, J2000, and diameter, 2 arcmin, for NGC 6863 but no other data are provided. Dias et al. (2002) includes NGC 6863 as a well established open cluster with coordinates, $\alpha=20^{\mathrm{h}} 05^{\mathrm{m}} 07^{\mathrm{s}}, \delta=-03^{\circ} 33^{\prime} 20^{\prime \prime}, l=38^{\circ} .2778$, $b=-17.9962$, J2000, and diameter, 3 arcmin, located 1200 pc from the Sun and with an age of 3.47 Gyr. SIMBAD 3 also classifies NGC 6863 as cluster of stars and refers to Bica et al. (2001). Conversely, the NGC/IC Project ${ }^{4}$ indicates that NGC 6863 is an asterism made of 8 stars.

An extensive search across published literature on this object reveals only three entries, all of them within the context of the Open Cluster Remnant paradigm. Bica et al. (2001) included NGC 6863 in their list of dissolving star cluster candidates with Galactic coordinates $l=38^{\circ} .27, b=-17.99, E(B-V)=0.28$, and age 3-4 Gyr. Pavani et al. (2007) presented preliminary spectroscopy for NGC 6863. Finally and using Two Micron All Sky Survey (2MASS, Skrutskie et al. 1997) and UCAC2 (Zacharias et al. 2004) data, Pavani \& Bica (2007) studied the structure, proper motions, and CMD distribution of this object to conclude that NGC 6863 is an open cluster remnant of old

\footnotetext{
1 http://www . univie.ac.at/webda/

2 http://www .astro.iag.usp.br/ wilton/

3 http://simbad.u-strasbg.fr/simbad

4 http://www.ngcicproject.org
} 
Table 1. Journal of photometric observations in the field of NGC 6863.

\begin{tabular}{cccc}
\hline \hline Field & Filter & $\begin{array}{c}\text { Time integration } \\
(\mathrm{s})\end{array}$ & $\begin{array}{c}\text { Seeing } \\
\left({ }^{\prime \prime}\right)\end{array}$ \\
\hline NGC 6863 & $U$ & 15,300 & 1.8 \\
& $B$ & $2,5,90$ & 1.9 \\
& $V$ & $1,3,30,30$ & 1.7 \\
& $I$ & $1,5,30$ & 1.6 \\
\hline
\end{tabular}

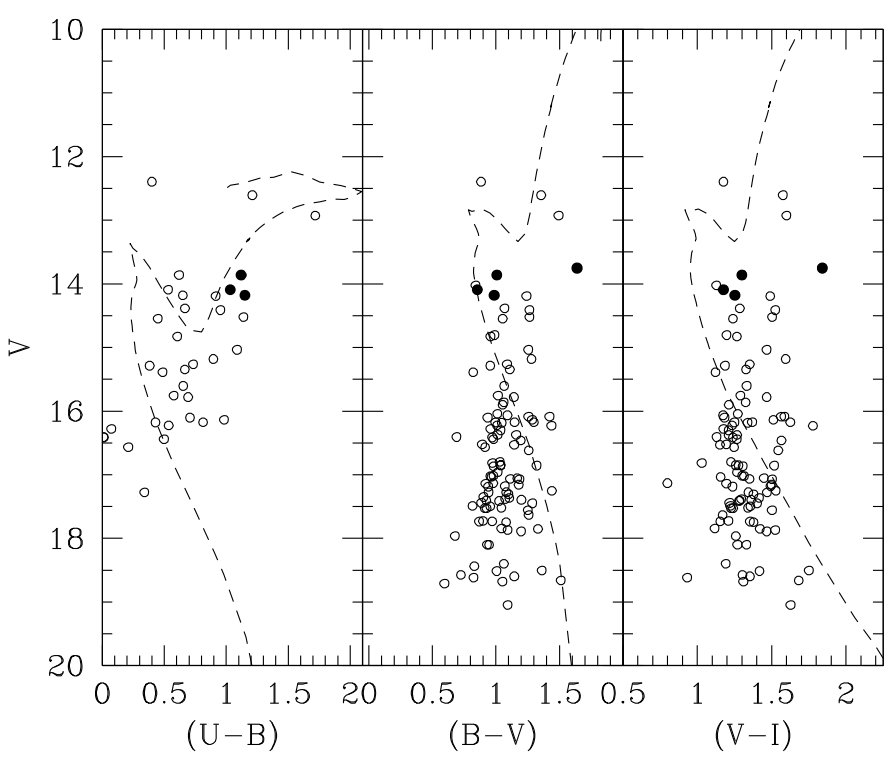

Fig. 2. CMDs in various color combinations for all the stars having UBVI photometry in the field of NGC 6863. Filled simbols indicate the 4 stars which identify the over-density (see Fig. 1). Super-imposed are a solar metallicity isochrone from Girardi et al. (2000) adopting the set of parameters derived by Pavani et al. (2007) for this asterism.

age, $3.5 \pm 0.5 \mathrm{Gyr}$, its centre and characteristic size as in Dias (2009), see above. An obvious over-density of bright stars can be seen in the central region of Fig. 1; however and following (e.g.) Odenkirchen \& Soubiran (2002), an overdensity of stars does not necessarily imply the existence of a physical ensemble.

\section{Observations and reduction}

Observations were performed in two different runs. The photometric one was carried out at Asiago (Italy) on December 2001, whereas the spectroscopic run was completed at ESO La Silla on September 2008. Details of both runs are given in the following sections.

\subsection{Photometry}

Observations were carried out with the AFOSC camera at the $1.82 \mathrm{~m}$ Copernico telescope of Cima Ekar (Asiago, Italy), in the photometric nights of December 17 and 18, 2001. AFOSC samples a $8.14 \times 8$ '.14 field in a $1 K \times 1 K$ thinned CCD. The typical seeing was between 1.8 and 2.3 arcsec. The details of the observations and data reductions are provided in Villanova et al. (2004a), where we reported on NGC 5385, Collinder 21 and NGC 2664, which were observed in the same run. Basic information on the NGC 6863 photometric run is given in Table 1.

From our photometric data we construct color-magnitude diagrams (CMD) for several color combinations. They are shown in Fig. 2. Here and with filled symbols we indicate the stars defining the asterism at the centre of the NGC 6863 field. These four stars produce the appearance of an overdensity in DSS images. All of them have $U B V I$ measurements with the exception of star \#4, which does not have $U$. The CMDs for the detected stars clearly do not show any distinctive feature that can lead us to think about a group of stars with common properties, a physical ensemble as in the case of, e.g., NGC 1901 discussed in Carraro et al. (2007). To guide the eye, and better clarify our conclusion, we over-impose in the same figure a solar metallicity isochrone taken from the Padova database (Girardi et al. 2000), and adopting the set of parameters from Pavani et al. (2007): 3.5 Gyr for the age, $E(B-V)=0.28$, and $(m-M)_{0} \sim 10.0$.

Our photometric material simply suggests that what we see in the direction of NGC 6863 is nothing but random Galactic field.

\subsection{Spectroscopy}

The spectra of the four brightest stars in NGC 6863 were collected at La Silla Observatory on 2008, September 18, with the HARPS fibre-fed spectrograph at the Cassegrain focus of the $3.6 \mathrm{~m}$ telescope. Exposure times varied between $1800 \mathrm{~s}$ and $3600 \mathrm{~s}$ depending on target brightness, and the resulting signal-to-noise ratio for each spectrum is given in Col. 2 of Table 2 . The light contribution from sky background was not negligible for our faint targets, and we allocated the second fiber to the sky instead of the simultaneous wavelength-calibration lamp, thus relying for calibration only on lamp frames acquired during daytime operations. However, the very high spectral resolution and instrumental stability guaranteed an accuracy still much higher than what was required for our aims.

We first attempted a manual data reduction by means of standard IRAF ${ }^{5}$ routines, comprising all steps from bias and flat-field corrections to spectra extraction and wavelength calibration. The inspection of the results revealed that there was no improvement, in terms of $\mathrm{S} / \mathrm{N}$ and spectral quality, with respect to the output of the online pipeline at the telescope. The wavelength calibration was also equivalent within calibration errors, and no systematic effect was detected. Hence, we adopted the pipeline-reduced, extracted and calibrated spectra. The extracted sky spectrum from the second fiber was subtracted to each target. The order \#46 of the second fiber falls between the two CCDs, and the corresponding science order was also suppressed, generating a gap between 5258 and $5336 \AA$. Spectra were convolved with a Gaussian filter to decrease the resolution to about $R=38000$, then re-binned in blocks of 4 pixels and re-sampled to a constant wavelength step of $0.01 \AA$. We thus increased the $\mathrm{S} / \mathrm{N}$ by a factor of two, while still keeping the resolution required for precise radial velocity and abundances measurements. Resulting spectra were divided order-by-order by the blaze function obtained by the pipeline from flat field frames, and any residual curvature of orders was eliminated fitting the continuum with a second-order polynomial. The orders thus normalized were finally merged to produce the final spectra. A section of the final spectra across the strong NaI doublet (5890-5893 $\AA$ ) is presented in Fig. 3 . The whole useful spectral range goes from 4000 to $6800 \AA$.

\footnotetext{
5 IRAF is distributed by the National Optical Astronomy Observatories, which are operated by the Association of Universities for Research in Astronomy, Inc., under cooperative agreement with the National Science Foundation.
} 
Table 2. Stellar data derived with spectroscopic analysis.

\begin{tabular}{ccccccccc}
\hline \hline ID & $\frac{S}{N}$ & $\begin{array}{c}T_{\text {eff }} \\
\mathrm{K}\end{array}$ & $\begin{array}{c}\log g \\
\mathrm{dex}\end{array}$ & $\begin{array}{c}{\left[\frac{\mathrm{Fe}}{\mathrm{H}}\right]} \\
\mathrm{dex}\end{array}$ & $\begin{array}{c}v_{\mathrm{t}} \\
\mathrm{km} \mathrm{s}^{-1}\end{array}$ & $\begin{array}{c}\mathrm{RV} \\
\mathrm{km} \mathrm{s}^{-1}\end{array}$ & $\begin{array}{c}E(B-V) \\
\mathrm{mag}\end{array}$ & $\begin{array}{c}d \\
\mathrm{pc}\end{array}$ \\
\hline 4 & 25 & $4110 \pm 65$ & $1.05 \pm 0.20$ & $-0.62 \pm 0.08$ & $1.28 \pm 0.20$ & $-55.1 \pm 0.3$ & $0.23 \pm 0.05$ & $6800 \pm 1100$ \\
5 & 15 & $5440 \pm 65$ & $3.55 \pm 0.20$ & $-0.09 \pm 0.08$ & $0.65 \pm 0.20$ & $21.6 \pm 0.5$ & $0.30 \pm 0.05$ & $1270 \pm 280$ \\
7 & 10 & $6100 \pm 75$ & $4.45 \pm 0.20$ & $-0.06 \pm 0.09$ & $1.32 \pm 0.15$ & $35.3 \pm 0.4$ & $0.34 \pm 0.04$ & $560 \pm 50$ \\
8 & 10 & $5320 \pm 65$ & $3.85 \pm 0.10$ & $-0.41 \pm 0.10$ & $1.24 \pm 0.15$ & $-10.6 \pm 0.5$ & $0.26 \pm 0.05$ & $740 \pm 90$ \\
\hline
\end{tabular}

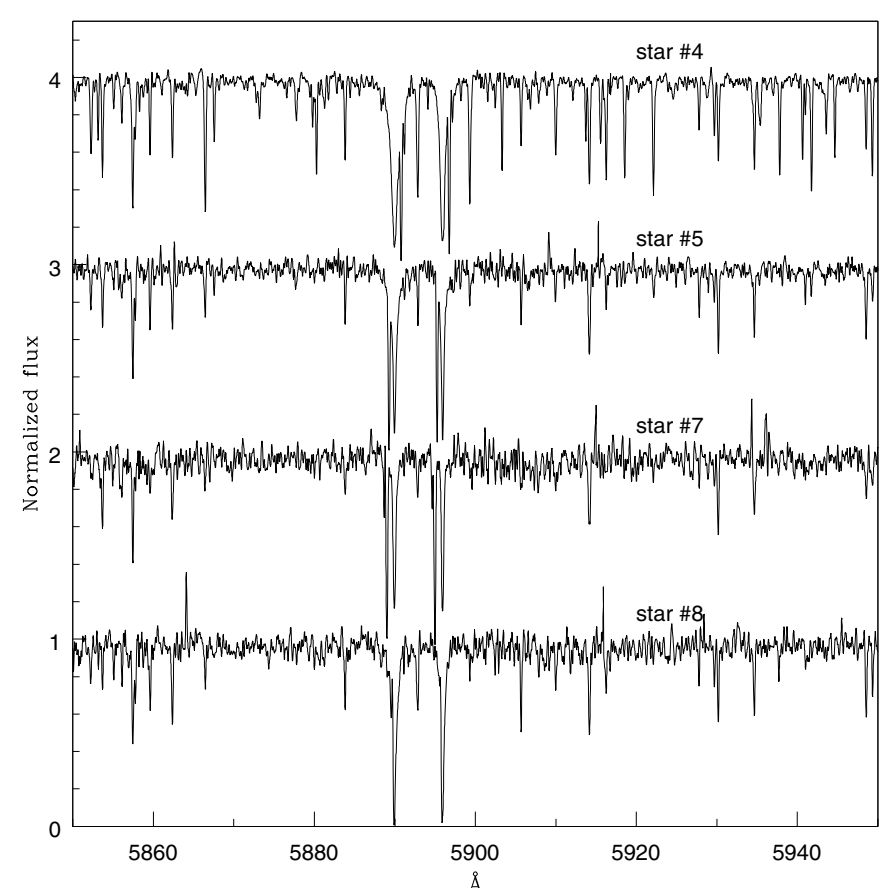

Fig. 3. A portion of the collected spectra, centred on the strong NaI doublet. An integer constant have been added to shift spectra vertically.

\subsubsection{Radial velocities}

Radial velocity (RV) measurements were performed with a cross-correlation (CC) technique (Tonry \& Davis 1979) using the fxcor IRAF task. As template we used a synthetic spectrum of solar metallicity drawn from the library of Coelho et al. (2005), with temperature and surface gravity intermediate between the values expected for program stars (4750 K and $2.0 \mathrm{dex}$, respectively). We verified through repeated measurements that different assumptions on template parameters in the range $T_{\text {eff }}=4000-5500 \mathrm{~K}$ and $\log g=1.0-3.0$ caused negligible changes in the resulting velocities $\left(\leq 0.2 \mathrm{~km} \mathrm{~s}^{-1}\right)$. The whole useful spectral range was used in the $\mathrm{CC}$, excluding the gap at $5300 \mathrm{~K}$ and the head of the telluric band at the red end of the range. In order to fix the zero-point of the measurements, this band was cross-correlated with a twilight solar spectrum acquired during a different run. Within $0.1 \mathrm{~km} \mathrm{~s}^{-1}$ the $\mathrm{CC}$ returned the solar RV at the dates of observations ${ }^{6}$, indicating that no correction for systematic effects was required. Finally, observed RVs were corrected to heliocentric velocities. Errors have been estimated as the quadratic sum of the most relevant sources, i.e. CC error $\left(0.2-0.4 \mathrm{~km} \mathrm{~s}^{-1}\right)$, the uncertainty in zeropoint definition $\left(0.1 \mathrm{~km} \mathrm{~s}^{-1}\right)$, and choice of the synthetic template $\left(0.2 \mathrm{~km} \mathrm{~s}^{-1}\right)$. Results are given in Col. 7 of Table 2 .

\footnotetext{
${ }^{6}$ http://eclipse.gsfc.nasa.gov/TYPE/TYPE.html
}

\subsubsection{Atmospheric parameters}

Effective temperature $\left(T_{\text {eff }}\right)$, surface gravity $(\log g)$, and metallicity of stars \#4 and \#5 were measured with the ionization and excitation balance method. In brief, equivalent widths (EWs) of a great number of FeI and FeII lines are measured, then $T_{\text {eff }}$ and microturbulent velocity $\left(v_{t}\right)$ are determined imposing that FeI abundances $(\epsilon(\mathrm{FeI}))$ of each individual line show no trend with excitation potential (EP) and EW, respectively, while $\log g$ is fixed by the requirement that FeI and FeII lines return the same iron abundance. We adopted the general iron line list of Moni Bidin (2009), which comprises atomic parameters for about 180 FeI and 40 FeII lines, with solar oscillator strengths and a lineby-line definition of the correction term to the Unsöld (1955) approximation of the collisional line broadening. However, we rejected the measurement when the line fit was not satisfactory or the stellar continuum was not easily defined, and only $\sim 70$ iron lines were finally used for each star. EWs were measured with a Gaussian fit with a dedicated IRAF script. Iron abundances were calculated with the LTE code MOOG $^{7}$ (Sneden 1973), with model atmospheres obtained interpolating from the Kurucz (1992) grid. The results are given in Table 2. We adopt the error estimates of Moni Bidin (2009), who compared the results with literature values for thirteen stars with multiple previous measurements. However, we improved the problematic fit of FeII lines, found at shorter wavelengths and affected by heavy line crowding, by simultaneously fitting all features in a region centred on the line of interest. Measurements on some test stars indicate that the scatter of $\log g$ is thus reduced, and we adopt here a corresponding lower error.

EW measurement for stars \#7 and \#8 was possible for too few lines $(\leq 30)$ and with large uncertainties, because of the low S/N. Consequently, the atmospheric parameter determination failed. For example, for star \#7 there was no physically meaningful combination of parameters that permitted to zero the trend of $\epsilon(\mathrm{FeI})$ with EWs and EP simultaneously. We therefore elaborated a different strategy. The wings of Balmer lines are a good indicator of temperature in the range $T_{\text {eff }}=5000-6500 \mathrm{~K}$ (Fuhrmann et al. 1994), and an explorative inspection of synthetic spectra revealed that they are quite insensitive to $\log g$. We fitted the wings of $\mathrm{H}_{\alpha}$ on target spectra with synthetic templates at different temperatures in steps of $250 \mathrm{~K}$, and results were fitted using a second-order polynomial to determine the minimum of the $\chi^{2}-T_{\text {eff }}$ relation. The estimate was repeated within a grid of values of metallicity and gravity $(\log g$ from 1 to $4,[\mathrm{Fe} / \mathrm{H}]=0$ and -0.5$)$ to check the extent of systematics, but only small random differences were found. We will assume the mean of these repeated measurements as the final result, and their rms as the associated error. We first tested the procedure on star \#1, obtaining $5400 \pm 65 \mathrm{~K}$. The excellent agreement with our estimate obtained with the EW method indicates that the results are reliable. It was not possible to repeat the test on star \#4, because Balmer lines rapidly vanish at decreasing temperature, and their reduced

\footnotetext{
${ }^{7}$ Freely distributed by C. Sneden, University of Texas, Austin.
} 


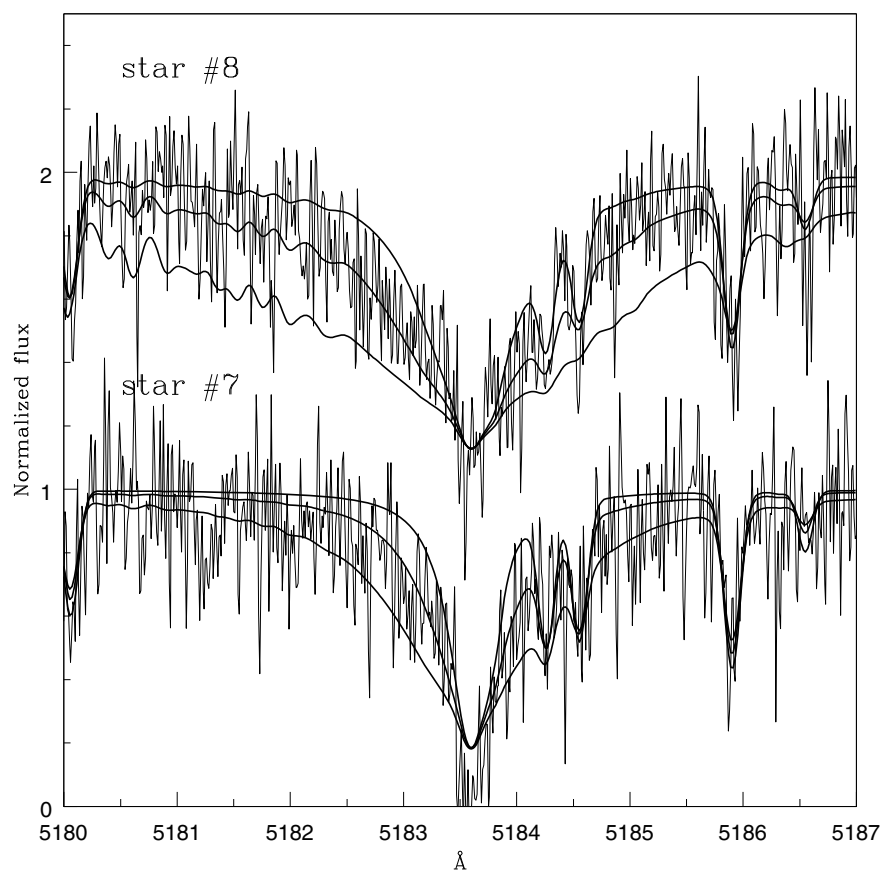

Fig. 4. The MgI line of stars \#7 and \#8, with overplotted (solid lines) synthetic profiles of templates with corresponding temperatures. The plotted models are for $\log g=3.0$ (narrower, upper profiles), $\log g=4.0$ (middle lines), and $\log g=5.0$ (wider, lower profiles). The spectra of star \#8 was vertically shifted adding a unit constant.

wings become insensitive to temperature changes. In fact, for this cool star the $\chi^{2}-T_{\text {eff }}$ was flat below about $4300 \mathrm{~K}$.

A similar routine was prepared to estimate $\log g$, fitting the wings of three gravity-sensitive lines: the CaI line at $6162 \AA$ (Edvardsson 1988, Katz et al. 2003), the redder line of the MgIb triplet at $5182 \AA$ (Kuijken et al. 1989), and the NaI doublet at 5890-5893 $\AA$. The temperature was fixed to the previouslydetermined value, interpolating the spectra using the synthetic library of Coelho et al. (2005), while both solar metallicity and $[\mathrm{Fe} / \mathrm{H}]=-0.5$ were used. The three lines were fitted independently, resulting in six gravity measurements per star. For stars $\# 4$ and \#5 we obtained $\log g=1.00 \pm 0.10$ and $3.70 \pm 0.10$ respectively, confirming again the reliability of the results. In Fig. 4 the MgI line of stars \#7 and \#8 is shown. As it can be seen, synthetic line profiles change noticeably with gravity, and a rough estimate of stellar gravity can even be done by visual inspection.

In order to fix the microturbulence velocity, we considered the equations available in the literature that estimate this parameter as a function of $T_{\text {eff }}$ and $\log g$. For star \#7 our choice fell on the relation presented by Reddy et al. (2003), that was calibrated using stars of the same spectral type as the target and presents a small quoted error $\left(0.15 \mathrm{~km} \mathrm{~s}^{-1}\right)$. Star \#8 is cooler than the temperature range where this equation was calibrated. The relation proposed by Gratton et al. (1996) returns a much lower $v_{\mathrm{t}}$ for cool stars, but it was calibrated on a mix of dwarfs and giants in a large range of temperatures, and the quoted uncertainty is much larger $\left(0.30 \mathrm{~km} \mathrm{~s}^{-1}\right)$. As no approach was fully satisfactory because both equations had a characteristic weakness, we decided to adopt the mean value as the best estimate of $v_{\mathrm{t}}$ for star \#8. However, different assumptions have only limited effects, because both relations return a metallicity within $1 \sigma$ of the final estimate.
The metallicity of stars \#7 and \#8 was measured from the EWs of the few iron lines for which a reliable fit was possible. The number of available lines was small (about 15), but their fit was good and the atmospheric parameters had already been determined. The rms of $[\mathrm{Fe} / \mathrm{H}]$ from single lines was about $0.15 \mathrm{dex}$, resulting in a formal error on the mean of about 0.04 dex. This was quadratically summed to the variations of $[\mathrm{Fe} / \mathrm{H}]$ induced by an increase/decrease of one parameter $\left(T_{\text {eff }}\right.$, $\log g, v_{t}$ ) by $1 \sigma$.

\subsubsection{Reddening and distances}

We finally compared the position of program stars in the $T_{\text {eff }}$ $\log g$ plane with Yale-Yonsei isochrones (Yi et al. 2003) of correct metallicity. The star position in this plane does not depend on reddening or distance, but it can depend on age. For each star we adopted the isochrone that matched the stellar parameters. However, this gives a reliable age estimate only for subgiant stars (\#5 and \#8), while at the tip of the RGB (star \#4) and in the MS (\#7) age is ill-defined because isochrones are too closely spaced. For stars \#5 and \#8 we obtained an age estimate of $2.3 \pm 1.0$ and $12 \pm 3$ Gyr respectively, where the errors were defined by the most extreme isochrones compatible with the $1 \sigma$ errors in temperature and gravity. We calculated the color excess $E(V-I)$ by comparison of observed and theoretical colors. $E(V-I)$ was then translated into $E(B-V)$ using the relations found by Cardelli et al. (1989). The two estimates of reddening usually differed because of observational errors, hence we adopted as final value their weighted mean with associated error. Results are presented in Col. 8 of Table 2. Uncertainties on color excesses were calculated from propagation of errors on observed and intrinsic colors, the latter being estimated as the variation induced by a change of $T_{\text {eff }}$ by $\pm 1 \sigma$. In fact, we verified that the intrinsic color is minimally affected by the errors on the other parameters $(\log g,[\mathrm{Fe} / \mathrm{H}]$ and age $)$. All but one of the values are very similar, and the mean reddening is $E(B-V)=0.28$. The extinction map of Schlegel et al. (1998) returns $E(B-V)=0.295$ in the direction of NGC 6863, in excellent agreement with our estimate. This also confirms that the temperatures adopted for program stars are substantially correct.

From isochrones we also estimated the absolute magnitude of each object, and we calculated the distance assuming $E(B-V)=0.28$ and $A_{\mathrm{V}}=3.1 E(B-V)$. The error on $M_{V}$ was assumed as the quadratic sum of the differences induced by the variation of each parameter $\left(T_{\text {eff }}, \log g,[\mathrm{Fe} / \mathrm{H}]\right.$ and age $)$ by $1 \sigma$, and the uncertainty on distance was calculated from propagation of errors. Results are presented in Col. 9 of Table 2. It is worth noticing that the distance of the MS star \#7 is well-defined, while errors on surface gravity induce large uncertainties on the distance of subgiants (\#5 and \#8).

It is interesting to note that our distance and age for star \#5 agree well with the values cited by Dias (2009) for NGC 6863 (1200 pc, $3.47 \mathrm{Gyr}$ ), but this is true for this star only.

\subsubsection{Spectroscopic results: discussion}

Metallicity, distance and radial velocity of star \#4 clearly show that it is a distant background star without any connection with the others. At a height $z=2.1 \mathrm{kpc}$ from the Galactic plane and with intermediate metallicity, it appears as a typical thick disk red giant. To a lesser extent, a similar conclusion can be drawn for star \#8 also. Its properties are less extreme, but its RV is very different from those of the two remaining stars, and its 


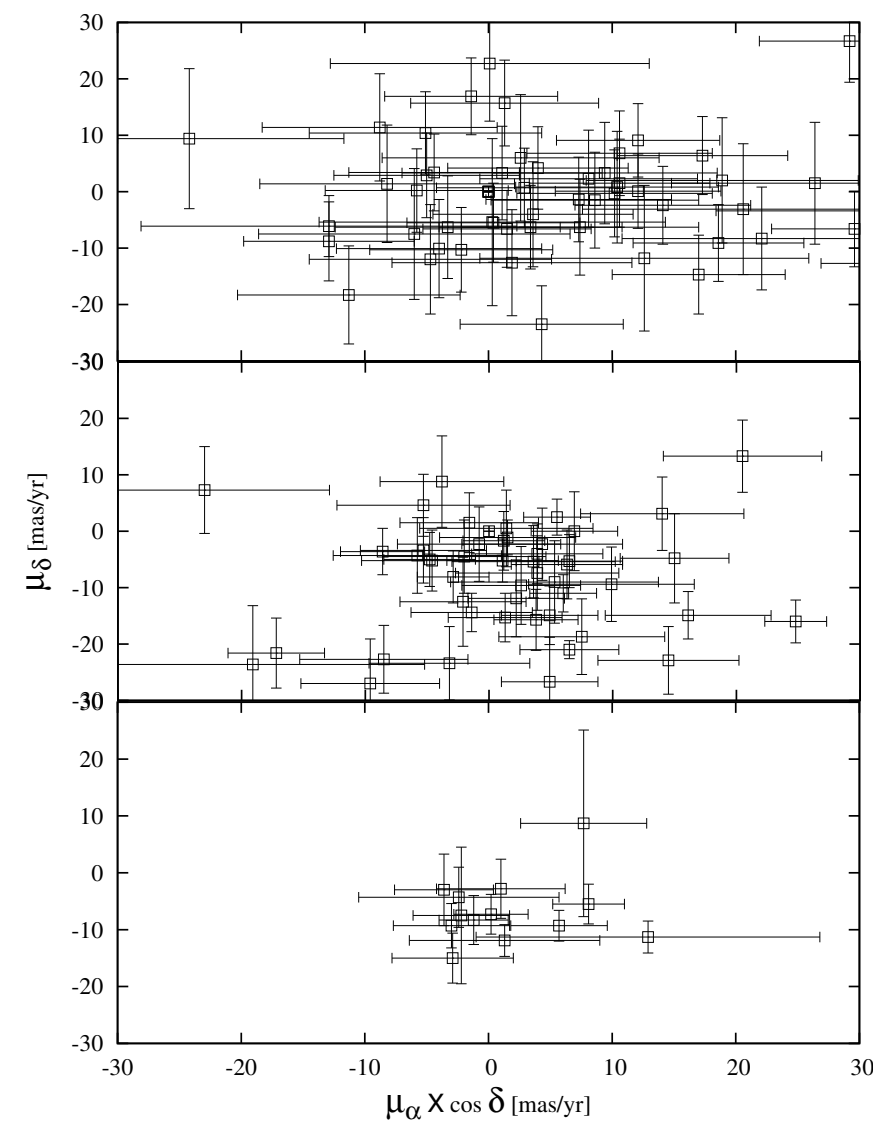

Fig. 5. Extended proper motion analysis. Top panel: stars having $12 \leq$ $K<14$. Middle panel: stars having $10 \leq K<12$. Bottom panel: stars brighter than $K=10$.

metallicity is lower by more than $3 \sigma$. Stars \#5 and \#7, on the other hand, have very similar metallicity. Their RVs differ by about $14 \mathrm{~km} \mathrm{~s}^{-1}$, but this fact alone does not exclude that the two objects can be part of a stellar system, because they were observed in a single epoch only, and one or both could be binaries. It is worth pointing out that indeed a very high binary fraction is expected in OCRs (de la Fuente Marcos 1997, 1998), and among the 13confirmed members of NGC 1901, to date the only spectroscopically-confirmed OCR, Carraro et al. (2007) measured at any epoch an internal RV dispersion between 16 and $20 \mathrm{~km} \mathrm{~s}^{-1}$. However, the spectroscopic distance of stars \#5 and \#7 still differ by $2.5 \sigma$. This is also consistent with the fact that there is no isochrone with $[\mathrm{Fe} / \mathrm{H}]=-0.08$ and $E(B-V)=0.28$ that fits the stellar position of both stars in the CMD. This difference excludes the possibility that they are part of the same stellar system. As a conclusion, the spectroscopic analysis reveals that the four brightest stars of NGC 6863 are not physically related; they are just a random alignment along the line of sight. In other words, they are an asterism.

\section{Proper motions}

Following Carraro et al. (2007), we extracted the proper motion components in a 20-squared arcmin field around NGC 6863 from the UCAC3 catalogue (Zacharias et al. 2009), and constructed 3 vector-point diagrams as a function of the $K$ magnitude (UCAC3 is cross-correlated with 2MASS). The results are shown in Fig. 5, where the bottom panel is restricted to stars having $K<10$, the middle panel to stars in the range $10 \leq K<12$, and the top panel to stars having $12 \leq K<14$. Stars in

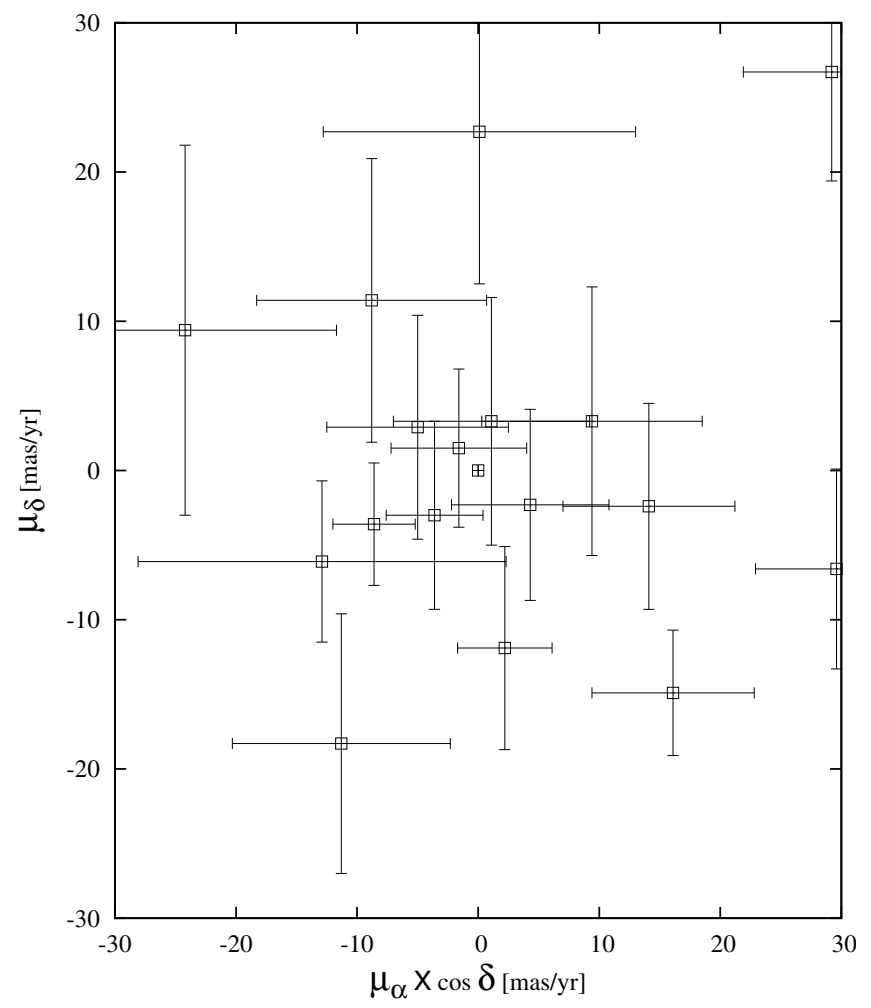

Fig. 6. Proper motion analysis. UCAC3 stars within 3 arcmin from the centre of the object.

Table 3. Proper motion data for the brightest stars.

\begin{tabular}{ccccc}
\hline \hline ID & $\begin{array}{c}\alpha(\mathrm{J} 2000) \\
(\mathrm{hms})\end{array}$ & $\begin{array}{c}\delta(\mathrm{J} 2000) \\
\left({ }^{\circ}{ }^{\prime \prime \prime}\right)\end{array}$ & $\begin{array}{c}\mu_{\alpha} \mathrm{UCAC} 3 \\
{[\mathrm{mas} / \mathrm{yr}]}\end{array}$ & $\begin{array}{c}\mu_{\delta} \mathrm{UCAC} 3 \\
{[\mathrm{mas} / \mathrm{yr}]}\end{array}$ \\
\hline 4 & $20: 05: 08.50$ & $-03: 33: 22.7$ & $2.2 \pm 3.9$ & $-11.9 \pm 6.8$ \\
5 & $20: 05: 07.67$ & $-03: 32: 59.3$ & $-8.6 \pm 3.4$ & $-3.6 \pm 4.1$ \\
7 & $20: 05: 06.07$ & $-03: 33: 29.3$ & $-12.9 \pm 15.2$ & $-6.1 \pm 5.4$ \\
8 & $20: 05: 06.78$ & $-03: 33: 26.6$ & $-49.9 \pm 6.1$ & $35.5 \pm 6.0$ \\
\hline
\end{tabular}

the lower panel appear to clump around $\mu_{\alpha}=-2.0$ [mas/yr], $\mu_{\delta}=-7.5$ [mas/yr]. Identifiable, consistent clumps are also observed in the other two panels. Is this apparent clump the kinematic signature of NGC 6863 ?

Following Dias (2009) and restricting our analysis to the central region, 3 arcmin around the centre of the cluster, the results are rather different, see Fig. 6. Clumping, if any, is observed around $\mu_{\alpha}=-1.5$ [mas/yr], $\mu_{\delta}=1.5$ [mas/yr]. This diagram includes all the stars brighter than $K=14$ with UCAC3 data within 3 arcmin of the centre of the object. In principle, we are using data similar (they use UCAC2 not UCAC3) to those in Pavani \& Bica (2007) but a direct comparison is not possible as they do not provide a diagram to illustrate the modulus of the proper motion distribution for NGC 6863. They only indicate that its characteristic median velocity is the highest in their open cluster remnant sample, $137 \mathrm{~km} \mathrm{~s}^{-1}$. In any case, preliminary visual inspection of Fig. 6 appears to confirm that the stellar population located around the commonly accepted centre of NGC 6863 is kinematically heterogeneous which is incompatible with open cluster status. In the following section, this conclusion is explored by using a more detailed statistical analysis.

As for the four brightest stars discussed above, individual proper motions are shown in Table 3. Calculating the Galactic space velocity for these stars as described in Johnson \& Soderblom (1987) with UCAC3 data in the Heliocentric 

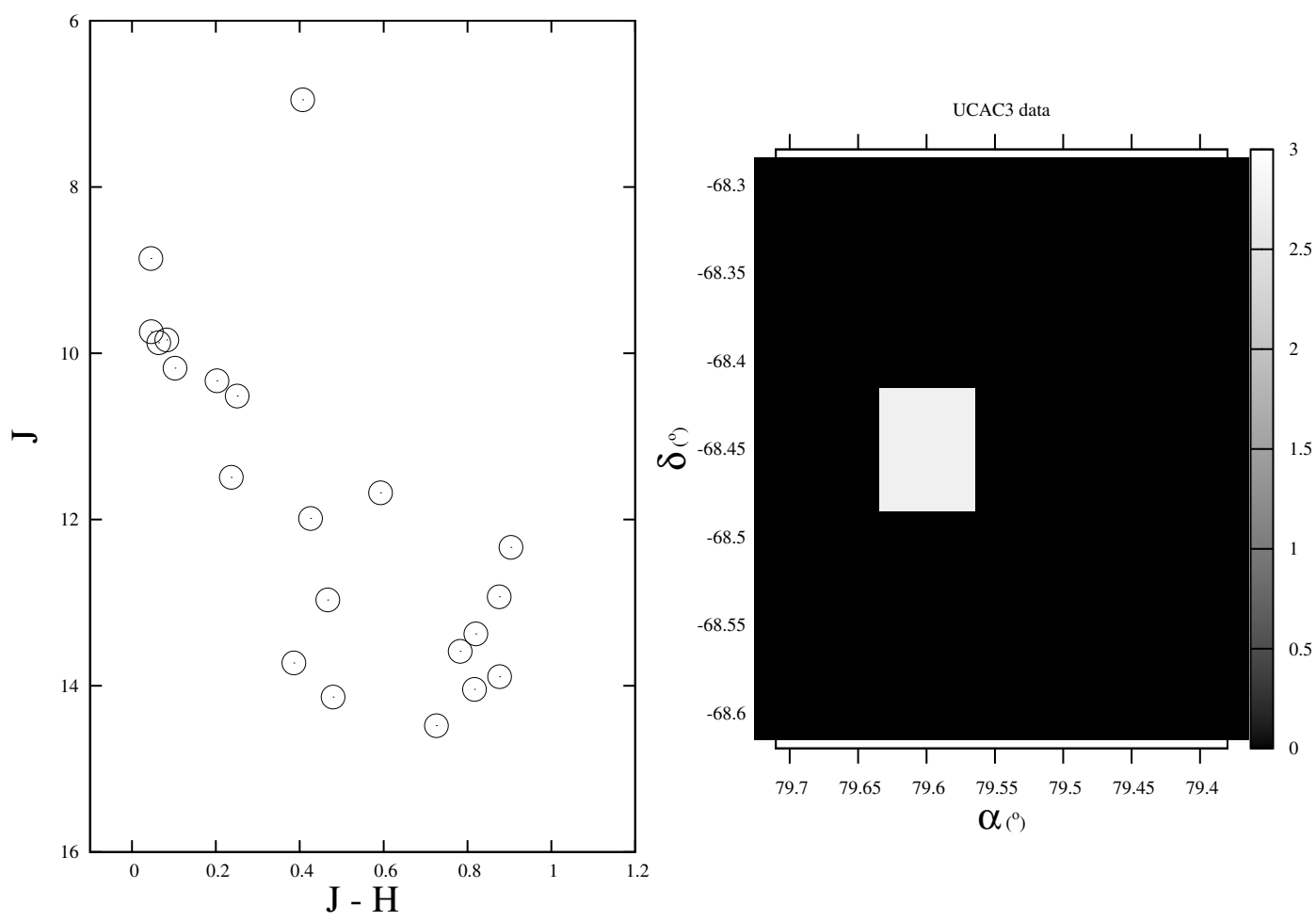

Fig. 7. NGC 1901. Left panel: 2 MASS CMD for those stars with proper motions in UCAC3 within $2 \sigma$ of $\left(\mu_{\alpha}, \mu_{\delta}\right)=(1.3 \pm 1.5,11.9 \pm 1.5)$ mas/yr. Right panel: Kulldorff's spatial scan statistics for the region around NGC $1901\left(20 \times 20 \operatorname{arcmin}^{2}\right.$ field, UCAC3 data). There is an obvious, statistically significant $(\sim 3 \sigma)$ over-density of stars with similar proper motions centred at the accepted coordinates for this OCR.

reference frame velocity $U V W$, we obtain results fully consistent with our previous spectroscopic analysis. For star \#4, $U=92 \mathrm{~km} \mathrm{~s}^{-1}, V=-320 \mathrm{~km} \mathrm{~s}^{-1}$, and $W=-211 \mathrm{~km} \mathrm{~s}^{-1}$, which is consistent with a halo star in a retrograde orbit. Star \#5 has $U=52 \mathrm{~km} \mathrm{~s}^{-1}, V=-14 \mathrm{~km} \mathrm{~s}^{-1}$, and $W=28 \mathrm{~km} \mathrm{~s}^{-1}$. Similar numbers are found for star \#7 with $U=51 \mathrm{~km} \mathrm{~s}^{-1}$, $V=2 \mathrm{~km} \mathrm{~s}^{-1}$, and $W=11 \mathrm{~km} \mathrm{~s}^{-1}$. Star \#8 is unusual and it could be a thick disk member with $U=25 \mathrm{~km} \mathrm{~s}^{-1}, V=58 \mathrm{~km} \mathrm{~s}^{-1}$, and $W=206 \mathrm{~km} \mathrm{~s}^{-1}$.

\section{An asterism in Aquila: statistical analysis}

At this point, one may want to know how significant, statistically speaking, is the kinematic evidence against NGC 6863 being a real open cluster. It is true that the four brightest stars in the field of NGC 6863 do not appear to form a physical system but what if just one of them is the brightest member of a real but poorly populated open cluster like NGC 1901 ? We are facing a spatial data mining problem where the goal is detection of a hypothetical over-density of kinematic nature (i.e. a small group of stars with similar proper motions) but in addition, statistical testing must be performed in order to determine whether the overdense regions are significant. In order to distinguish the clumps that are significant from those that are likely to have occurred by chance we use Kulldorff's spatial scan statistic ( $D_{\mathrm{K}}$ defined below, Kulldorff 1997). In the following, we assume data has been aggregated to an $N \times N$ grid of squares, where each grid cell is associated with a count $c_{i j}$ (number of stars with certain properties, proper motions in this case) and an underlying population $p_{i j}$, with $i, j=1, \ldots, N$. Our goal is to find over-densities: spatial regions where the counts are significantly higher than expected, given the underlying population. Our objective is to search over all square regions and find the region(s) with the highest density according to a density measure. Kulldorff's statistic assumes that counts $c_{i j}$ are generated by an inhomogeneous Poisson process with mean $q p_{i j}$, where $q$ is the underlying expected value of the count to population ratio. We then calculate the logarithm of the likelihood ratio of two possibilities: that $q$ is higher in the region than outside the region or that it is identical inside and outside the region. For a cell with count $c_{i j}$ and population $p_{i j}$, in a grid with total count $C$ and population $P$, we can calculate:

$D_{\mathrm{K} i j}=c_{i j} \log \frac{c_{i j}}{p_{i j}}+\left(C-c_{i j}\right) \log \frac{C-c_{i j}}{P-p_{i j}}-C \log \frac{C}{P}$

if $c_{i j} / p_{i j}>C / P$, and $D_{\mathrm{K} i j}=0$ otherwise. Kulldorff (1997) proved that the spatial scan statistic is individually more powerful for finding a single significant region of unusually high density than any other test statistic. Unfortunately, performance of scan statistics is affected by tessellation geometry (variation of cell size and cell shape) as well as aggregation level (average cell size). A single actual cluster that is bisected by a large cell may appear either as not significant or as two distinct clusters depending on whether the large cell is included in the candidate zone (Kulldorff et al. 2003). In the following analysis, special care has been taken to minimize tessellation effects by using multiple grid sizes and checking for consistency. On the other hand, we have calculated $D_{\mathrm{K} i j}$ for each cell in the grid, the standard deviation, and plot $\left(D_{\mathrm{K} i j}-\left\langle D_{\mathrm{K}}\right\rangle\right) / \sigma$ using colour maps. To test the reliability of our statistical approach we first apply it to a well established OCR, NGC 1901, and then to our current object, NGC 6863.

In order to carry out this analysis we use data from 2MASS. The 2MASS All Sky Catalog of Point Sources (Cutri et al. 2003) includes data for both NGC 1901 and NGC 6863 and $20 \times 20 \operatorname{arcmin}^{2}$ areas around the coordinates of the accepted centre of these objects were extracted. Following Pavani \& Bica (2007) we use $E(B-V)=3.03 \times E(J-H), A_{J} / A_{V}=0.282$ and $A_{J}=2.65 \times E(J-H)($ Rieke \& Lebofsky 1985). 

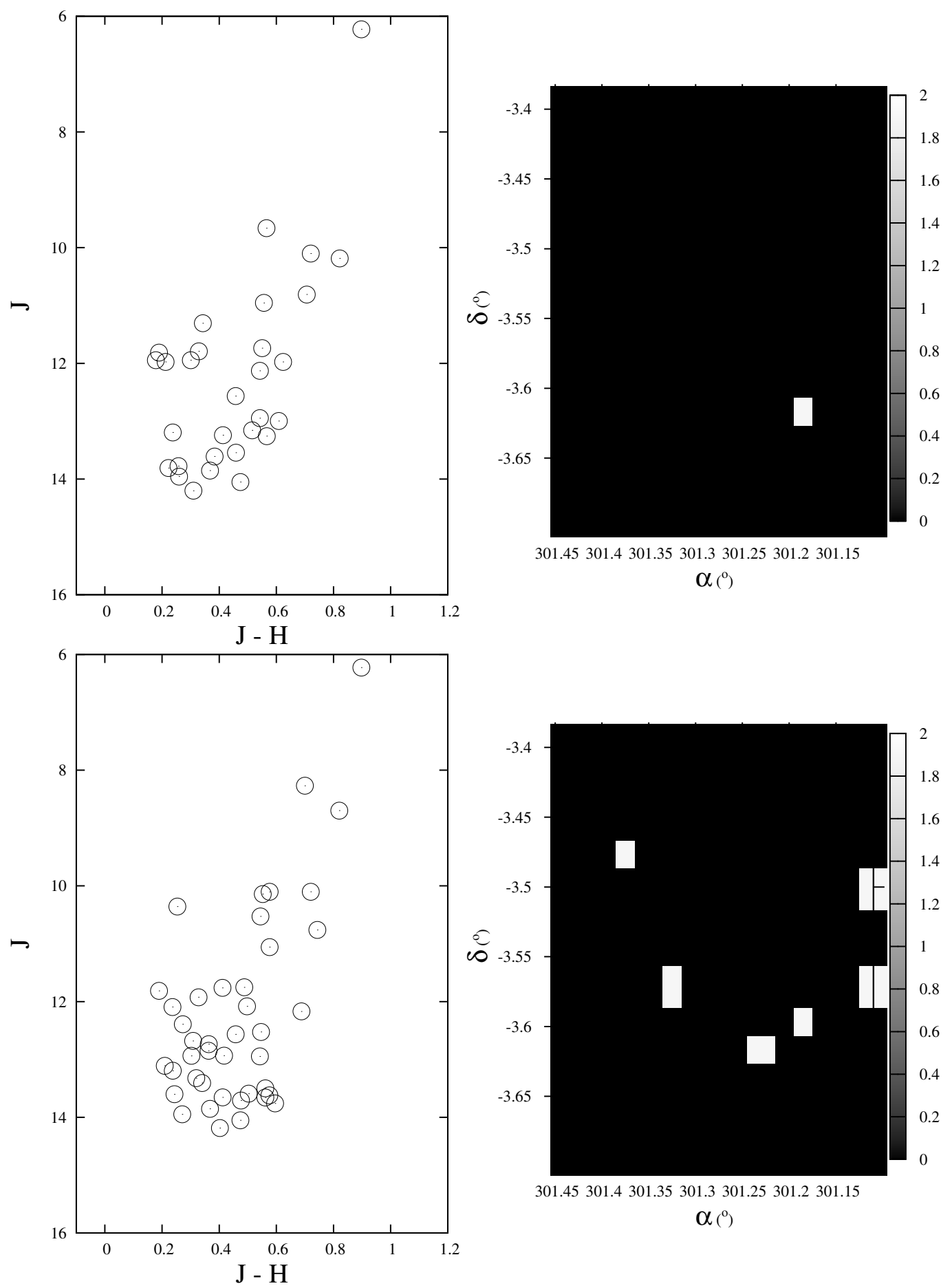

Fig. 8. Star \#4. Top left panel: 2MASS CMD for those stars with proper motions in UCAC3 within $1 \sigma$ of $\left(\mu_{\alpha}, \mu_{\delta}\right)=(2.2 \pm 3.9,-11.9 \pm 6.8)$ mas/yr. Top right panel: Kulldorff's spatial scan statistics for star \#4. No statistically significant clustering is visible in this plot. Star \#7. Bottom left panel: 2MASS CMD for those stars with proper motions in UCAC2 within $1 \sigma$ of $\left(\mu_{\alpha}, \mu_{\delta}\right)=(-12.9 \pm 15.2,-6.1 \pm 5.4)$ mas/yr. Bottom right panel: Kulldorff's spatial scan statistics for star \#7. There is no significant overdensity at the expected location of NGC 6863. As for stars \#5 and \#8, no overdensity of any kind is observed.

\subsection{NGC 1901}

Using UCAC2 data, Carraro et al. (2007) concluded that the average proper motions of this genuine OCR were $\left(\mu_{\alpha}, \mu_{\delta}\right)=(1.7 \pm$ $1.3,12.3 \pm 2.9) \mathrm{mas} / \mathrm{yr}$. Repeating the analysis with UCAC3 data for the suspected NGC 1901 members compiled in Table 2 of that paper we obtain $\left(\mu_{\alpha}, \mu_{\delta}\right)=(1.3 \pm 1.5,11.9 \pm 1.5) \mathrm{mas} / \mathrm{yr}$. This translates into $U=-22.4 \pm 8.4 \mathrm{~km} \mathrm{~s}^{-1}, V=-3.0 \pm 1.7 \mathrm{~km} \mathrm{~s}^{-1}$, and $W=-1.9 \pm 2.8 \mathrm{~km} \mathrm{~s}^{-1}$, which is consistent with other recent results (see Bovy et al. 2009, Fig. 21). If we apply the spatial scan statistics to the corresponding UCAC3 sample $(20 \times 20$ $\operatorname{arcmin}^{2}$ around the cluster centre) looking for over-densities within $2 \sigma$ of the average proper motions, we obtain the plot in Fig. 7 (right panel). The effective size of the OCR is $\sim 3$ arcmin (Carraro et al. 2007); this is the grid cell size used in the analysis. A statistically significant over-density, $\sim 3 \sigma$, appears at the expected position. The 2MASS CMD is also provided, $E(B-V)=0.04$ was used in the reduction process. The CMD 
in Fig. 7 is analogous to the one in Pavani \& Bica (2007) even if a slightly different $E(B-V)$ was used; see their Fig. 7, central panel. Photometry does show a defined main sequence and the CMD hints of all the characteristic properties of OCRs as predicted by $N$-body simulations (de la Fuente Marcos 1998): scarcity of low-mass members, high binary fraction (a double main sequence is not unlikely), and overall sparse population. These results together with the kinematically homogeneous signature confirm that the statistical analysis described above generates robust results. NGC 1901 is a true representative of the terminal stage in the life of an open cluster, the OCR phase. Its current properties are compatible with NGC 1901 being what remains of a relatively small cluster with an original population of 500-750 stars (Villanova et al. 2004b; Carraro et al. 2007).

\subsection{The NGC 6863 asterism}

If we repeat the same approach looking for over-densities associated to the four brightest stars in the field of NGC 6863 we obtain Fig. 8. Here, the effective size of the hypothetical OCR is $\sim 1.5$ arcmin (Pavani \& Bica 2007) and this is the grid cell size used in the analysis. $E(B-V)=0.28$ was used to plot the CMDs. No obvious over-density can be found at the location of NGC 6863. It is, therefore, reasonable to conclude that the actual presence of an open cluster, remnant or not, at the location of NGC 6863 is very unlikely. The application of the Kulldorf statistics shows that NGC 1901 is a real OCR, whereas it confirms that there is no evidence to suggest that NGC 6863 really exists.

\section{Conclusions}

In this paper we have presented detailed photometric, spectroscopic and kinematic evidence against the classification of NGC 6863 as open cluster. Our results show that the four brightest stars commonly associated to NGC 6863 form an asterism, a group of non-physically associated stars projected together. The spectra of the four brightest stars in this field clearly indicate that they are part of different populations. Their radial velocities are statistically very different and their spectroscopic parallaxes are inconsistent with them being part of a single, bound stellar system. Out of the four stars, only two of them have similar metallicity. As for the underlying open cluster remnant described in Pavani \& Bica (2007), our analysis of the existing data has demonstrated convincingly that there is no base whatsoever to consider NGC 6863 as a possible OCR. The color magnitude diagrams for the field of NGC 6863 do not even show any clear signature typical of actual open clusters. Spatial scan statistics using UCAC3 data strongly suggests that no statistically significant, kinematically supported over-density is present at the purported location of NGC 6863 although the proper motion errors are rather large. This together with the other issues discussed above force us to conclude that the presence of a star cluster of any kind associated to the coordinates of NGC 6863 is highly unlikely.

Acknowledgements. C.M.B. acknowledges the Chilean Centro de Excelencia en Astrofísica y Tecnologías Afines (CATA), and Francesco Mauro for very usefull discussions. G.C. deeply acknowledges the entire Asiago technical staff for the kind night assistance over the whole duration of this project. In preparation of this paper, we made use of the NASA Astrophysics Data System and the ASTRO-PH e-print server. This research has made use of the WEBDA database operated at the Institute of Astronomy of the University of Vienna, Austria. This work also made extensive use of the SIMBAD database, operated at the CDS, Strasbourg, France. This publication makes use of data products from the Two Micron All Sky Survey, which is a joint project of the University of Massachusetts and the Infrared Processing and Analysis Center/California Institute of Technology, funded by the National Aeronautics and Space Administration and the National Science Foundation.

\section{References}

Aarseth, S. J. 1968, Bull. Astron., Ser., 3, 105

Allison, M. 2006, Star Clusters and How to Observe Them (Springer-Verlag London Limited), 45

van Albada, T. S. 1968, Bull. Astron. Inst. Neth., 19, 479

Bica, E., Santiago, B. X., Dutra, C. M., et al. 2001, A\&A, 366, 827

Bovy, J., Hogg, D. W., \& Roweis, S. T. 2009, ApJ, 700, 1794

Cardelli, J. A., Clayton, G. C., \& Mathis, J. S. 1989, ApJ, 345, 245

Carraro, G. 2006, BASI, 34, 153

Carraro, G., Dinescu, D. I., Girard, T. M., \& van Altena, W. F. 2005, A\&A, 433, 143

Carraro, G., de La Fuente Marcos, R., Villanova, S., et al. 2007, A\&A, 466, 931

Coelho, P., Barbuy, B., Meléndez, J., Schiavon, R. P., \& Castilho, B. V. 2005, A\&A, 443, 735

Cutri, R., Skrutskie, M. F., Van Dyk, S., et al. 2003, The 2MASS All-Sky Catalog of Point Sources, University of Massachusetts and IPAC/California Institute of Technology

Dias, W. S., Alessi, B. S., Moitinho, A., \& Lépine, J. R. D. 2002, A\&A, 389, 871 Dreyer, J. L. E. 1888, Mem. R. Astron. Soc., 49, 1 (reprinted 1962, London: Royal Astronomical Society)

de la Fuente Marcos, R. 1997, A\&A, 322, 764

de la Fuente Marcos, R. 1998, A\&A, 333, L27

Edvardsson, B. 1988, A\&A, 190, 148

Fuhrmann, K., Axer, M., \& Gehren, T. 1994, A\&A, 285, 585

Girardi, L., Bertelli, G., Bressan, A., \& Chiosi, C. 2000, A\&AS, 141, 371

Gratton, R. G., Carretta, E., \& Castelli F. 1996, A\&A, 314, 191

Herschel, J. F. W. 1833, Phil. Tran. R. Soc., 123, 359

Herschel, J. F. W. 1864, Phil. Tran. R. Soc., 154, 1

von Hoerner, S. 1960, Z. Astrophys., 50, 184

von Hoerner, S. 1963, Z. Astrophys., 57, 47

Johnson, D. R. H., \& Soderblom, D. R. 1987, AJ, 93, 864

Katz, D., Farata, F., Aigrain, S., \& Micela, G. 2003, A\&A, 397, 747

Kuijken, K., \& Gilmore, G. 1989, MNRAS, 239, 605

Kulldorff, M. 1997, Commun. Statist.: Theory Meth., 26, 1481

Kulldorff, M., Tango, T., \& Park, P. 2003, Computational Statistics and Data Analysis, 42, 665

Kurucz, R. L. 1992, in The Stellar Population of Galaxies, ed. B. Barbuy, \& A. Renzini (Dordrecht Reidel), IAU Symp., 149, 225

Lada, C. J., \& Lada, E. A. 2003, ARA\&A, 41, 57

Lodén, L. O. 1987, Ir. Astron. J., 18, 95

Lodén, L. O. 1988, Ap\&SS, 142, 177

Lodén, L. O. 1993, Ap\&SS, 199, 165

Mermilliod, J.-C., \& Paunzen, E. 2003, A\&A, 410, 511

Moni Bidin, C. 2009, Ph.D. Thesis, Universidad de Chile, 1

Odenkirchen, M., \& Soubiran, C. 2002, A\&A, 383, 163

Pavani, D. B., \& Bica, E. 2007, A\&A, 468, 139

Pavani, D. B., Bica, E., Ahumada, A. V., \& Clariá, J. J. 2007, in Stellar Populations as Building Blocks of Galaxies, ed. A. Vazdekis, \& R. F. Peletier (Cambridge: Cambridge University Press), 256

Reddy, B. E., Tomkin, J., Lambert, D. L., \& Allende Prieto, C. 2003, MNRAS, 340,304

Rieke, G. H., \& Lebofsky, M. J. 1985, ApJ, 228, 618

Schlegel, D. J., Finkbeiner, D. P., \& Davis, M. 1998, ApJ, 500, 525

Skrutskie, M. F., et al. 1997, in The Impact of Large Scale Near-IR Sky Surveys (Dordrecht: Kluwer Academic Publishing Co.), ed. F. Garzon et al., 25

Sneden, C. 1973, ApJ, 184, 839

Sulentic, J. W., \& Tifft, W. G. 1973, The Revised New General Catalogue of Nonstellar Astronomical Objects (Tucson, Arizona: The University of Arizona Press)

Tonry, J., \& Davis, M. 1979, AJ, 84, 1511

Villanova, S., Carraro, G., de la Fuente Marcos, R., \& Stagni, R. 2004a, A\&A, 428, 67

Villanova, S., Carraro, G., \& de la Fuente Marcos, R., 2004b, Proc. ASP Conf., 317,196

Unsöld, A. 1955, Physik der Sternatmosphären, 2nd edn. (Berlin: Springer, Berlin, New York: Heibelberg)

Wielen, R. 1975, in Dynamics of Stellar Systems, ed. A. Hayli (Dordrecht: Reidel), 97

Yi, S. K., Kim, Y.-C., \& Demarque, P. 2003, ApJS, 144, 259

Zacharias, N., Urban, S. E., Zacharias, M. I., et al. 2004, AJ, 127, 3043

Zacharias, N., Finch, C., Girard, T., et al. 2009, AJ, in press 